\title{
Topical Review: Design, Fabrication, and Applications of Hybrid Nanostructured Array
}

\author{
Shaoli Zhu and Wei Zhou \\ School of Mechanical and Aerospace Engineering, Nanyang Technological University, 50 Nanyang Avenue, Singapore 639798 \\ Correspondence should be addressed to Shaoli Zhu, s.zhu2@uq.edu.au
}

Received 20 July 2011; Accepted 31 August 2011

Academic Editor: Hui Wang

Copyright (๑) 2012 S. Zhu and W. Zhou. This is an open access article distributed under the Creative Commons Attribution License, which permits unrestricted use, distribution, and reproduction in any medium, provided the original work is properly cited.

\begin{abstract}
Nanohybrid materials have been widely used in the material chemistry research areas. In this paper, we mainly discussed the hybrid nanostructures used for nanobiosensor applications. It is one of the most promising and rapidly emerging research areas in nanotechnology field. Design, fabrication, and applications of hybrid nanostructures are reviewed, respectively. Finite difference time domain (FDTD) methods are applied to design different materials of hybrid nanostructures. Nanosphere lithography (NSL) is used to fabricate our designed hybrid nanostructures. Moreover, protein A and staphylococcal enterotoixn B (SEB), an enterotoxin, are detected by our designed hybrid nanostructures. From all the experiment results, we can see that our designed hybrid nanostructures are one of important nanohybrid materials. They have many potential applications in the nanobiosensor in the future.
\end{abstract}

\section{Introduction}

Localized surface plasmon resonance- (LSPR-) based nanobiosensors are of great interest in various applications such as environmental protection [3-5], biotechnology [6-8], and food safety [8]. It is well known that LSPR can be excited when the incident photon frequency is resonant with the collective oscillation of the conduction electrons. It is named as the LSPR effect. Transmission peaks of LSPR-related spectra are sensitive to the electric medium on surface of metal films. The LSPR-based nano-biosensor is a refractive index-based sensing device which relies on the extraordinary optical properties of noble metal (e.g., $\mathrm{Ag}, \mathrm{Au}$, and $\mathrm{Cu}$, etc.) nanoparticles $[9,10]$. The sensing capability of the LSPR sensor can be modified by tuning shape, size, and material composition of the nanoparticles. The nanoparticles are effective for quantitative detection of chemical and biological targets [4]. The sensing principle employed in these experiments relies on the high sensitivity of the LSPR spectrum of the noble metal nanoparticles due to an absorbate-induced change occurring in the dielectric constant of the surrounding environment. The local environment that surrounds the nanoparticles can be modified by means of binding of the biological molecular. The extinction spectrum of the nanosensor can be derived using a spectrophotometer [11].

Many biological and chemical agents such as bacteria, algae, fungi, viruses, and toxins are capable of extensively affecting humans and animals [12-15]. Staphylococcus aureus enterotoxin $B(S E B)$, a small protein toxin $[12,16]$, was selected as a typical small protein toxin in our experiments. SEB with $28.4 \mathrm{kDa}$ protein toxin is one of a group of five major serological types of related proteins with molecular weights ranging from $26 \mathrm{kDa}$ to $29.6 \mathrm{kDa}$. SEB is an incapacitating toxin, but it is rarely lethal. Detection and quantification of SEB in buffer were demonstrated using the LSPR-based nano-biosensor. Theoretically, the detection concentration of SEB can reach to nanogramme per milliliter level. The methods used to detect SEB include enzymelinked immunosorbent assays (ELISAs) [17], light addressable potentiometric sensors (LAPSs) [18], array biosensors [19], immunomagnetic separation electro-chemiluminescence and fluorescence procedures (IMS-ECL and IMSFCL) [20] or rapid chromatographic assays (RCA) [21], and Surface Plasmon Resonance (SPR) sensors [22]. In our previous research work, we have designed and explored some nanohybrid materials to detect the different refractive index 


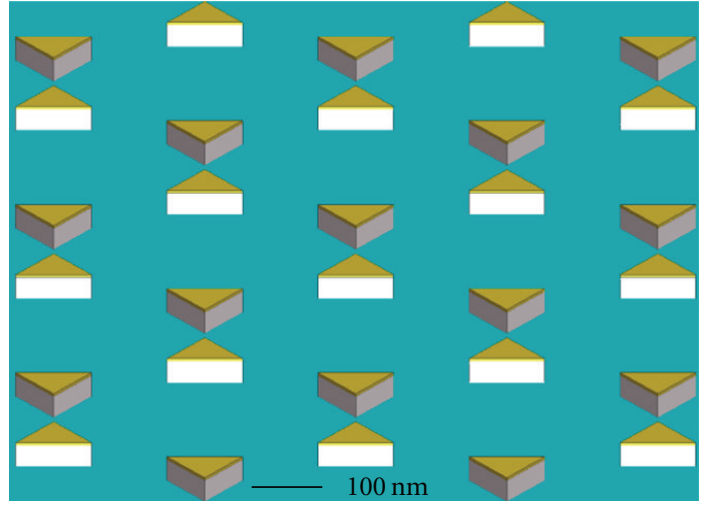

(a)

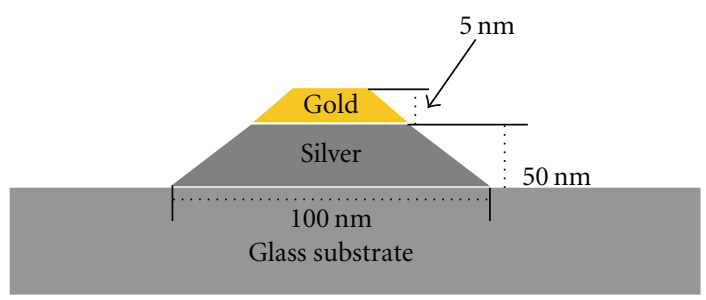

(b)

Figure 1: (a) Three-dimensional geometrical model of triangular hybrid Au-Ag nanostructures, (b) cross-section of a single hybrid $\mathrm{Au}-\mathrm{Ag}$ triangular nanostructures. From $[1,2]$.

of chemical materials and the various kinds of biomolecules $[1,2,23-27]$. In this paper, we reviewed the design, fabrication, and applications of hybrid nanostructured array. The paper is divided into the following three parts: (1) finitedifference and time-domain (FDTD) algorithm was used to design the hybrid nanostructured array. (2) Nanosphere lithography was employed to fabricate our designed array. (3) The functionalized nanostructures are used for detection of proteins A and SEB.

\section{Design Methods for Hybrid Nanostructures}

There are many design methods to aid in deciding the parameters of the nanostructured array. For example, Mie scattering theory [28] discrete dipole approximation (DDA) [29], finite integration technique (FIT) [30], finite difference time domain (FDTD) [31] method, and the finite element method [32] are widely used for the design of the nanoparticles. FDTD is a commercial software to design arbitrary shapes, sizes and periods of nanostructures. The following parts are our design model and method. The triangular hybrid $\mathrm{Au}-\mathrm{Ag}$ nanostructure array was proposed as a sensitive cell of the LSPR-based nano-biosensor. Using FDTD algorithm [33], we designed and calculated the refractive index sensitivity of the hybrid nanostructures. The corresponding model of the triangular hybrid Au-Ag nanostructure array is shown in Figure 1(a). A cross-section of a single particle labeling the materials of the substrate and particle and their thicknesses is shown in Figure 1(b).

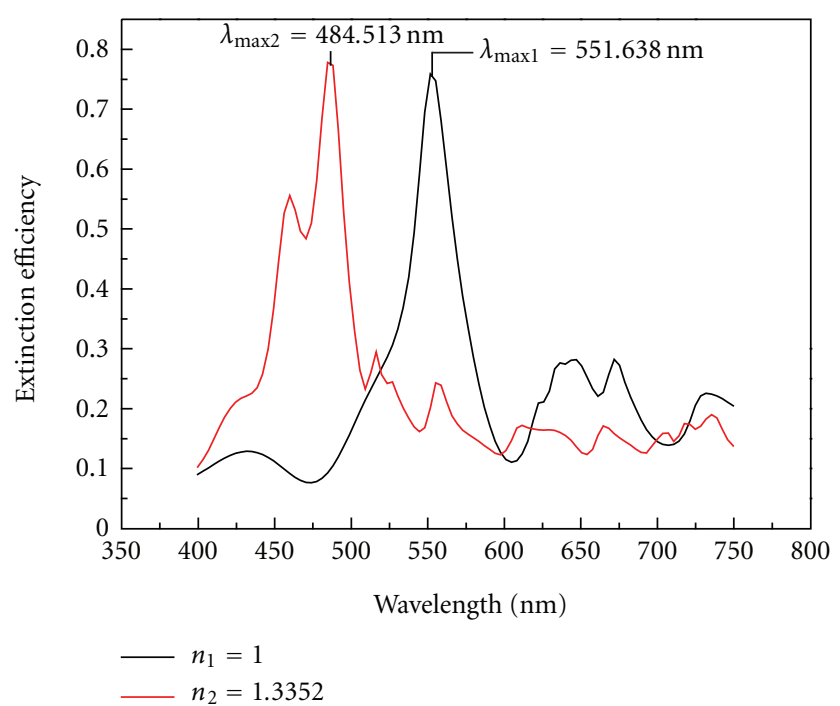

Figure 2: FDTD solution calculation results when the refractive index medium surrounding this hybrid nanostructures are 1.0 and 1.3352 , respectively. From [1].

The out-of plane heights of the Ag nanostructures under the Au layer is $50 \mathrm{~nm}$ and the top Au nanostructures is $5 \mathrm{~nm}$ only. The in-plane widths of each nanostructures are $100 \mathrm{~nm}$. The period of the nanostructure array is $400 \mathrm{~nm}$. In order to investigate the effectiveness of mediums with different refractive index surrounding the hybrid nanostructures, we selected the air $\left(n_{1}=1.0\right)$ and Protein A (Protein A: PBS $\left.(0.01 \mathrm{M}, \mathrm{pH}=7.4)=1: 100, n_{2}=1.3352\right)$ surrounding the nanostructures. When the refractive index of the mediums surrounding this hybrid nanostructures is 1.0 and 1.3352, respectively, the FDTD algorithm-based calculation results can be obtained, as shown in Figure 2. From the results, we can calculate the sensitivity of the hybrid $\mathrm{Au}-\mathrm{Ag}$ triangular nanostructure array as $S=\left(\lambda_{\max 1}-\lambda_{\max 2}\right) /\left(n_{2}-n_{1}\right)=$ $(551.638-484.513) /(1.3352-1.0)=200 \mathrm{~nm} / \mathrm{RIU}$. It can realize a detection of SEB with higher sensitivity.

\section{Fabrication of Nanostructures}

An NSL technique [34] was employed to create the surfaceconfined hybrid $\mathrm{Au}-\mathrm{Ag}$ triangular nanostructures supported on a glass substrate (see Figure 3). NSL process begins from the self-assembly of size-monodisperse nanospheres into a two-dimensional (2D) colloidal crystal. As the solvent of the nanosphere solution evaporates, capillary forces draw the nanospheres together, thereby crystallizing them into a hexagonally close-packed pattern on the substrate. Following self-assembly of the nanosphere mask, some silver and gold metals are deposited onto the nanosphere-coated substrate, respectively. Metal deposition parameters and vacuum condition are listed as follows: vacuum $2 \times e-6$ Torr, deposition rate $0.4 \mathrm{~A} / \mathrm{sec}$. After the metals deposition, the nanosphere mask is removed via sonication in ethanol resulting in surface-confined nanostructures with triangular footprints. The nanostructures have out-of plane heights of silver 


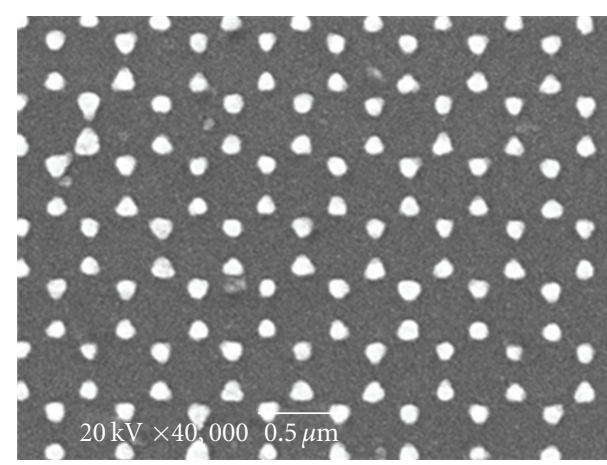

FIGURE 3: SEM image of topography of the triangular hybrid Au-Ag nanostructures fabricated by NSL. From [1].

nanostructures $\sim 50 \mathrm{~nm}$ and the upper gold nanostructures $\sim 5 \mathrm{~nm}$ in thickness, and $\sim 100 \mathrm{~nm}$ in plane widths of each nanostructure and $\sim 400 \mathrm{~nm}$ period of the nanostructure array as measured using JSM-5900 LA scanning electron microscope (SEM). In order to approve the existence of $\mathrm{Au}$ cap layer, we used EDX to analyse the materials of hybrid nanostructured array. Figure 4 shows the EDX results for all the elements for the hybrid nanostructured array. EDX analysis results are based on the same materials as the hybrid $\mathrm{Au}$ and $\mathrm{Ag}$ film. From these results, we can see that there are $\mathrm{Au}$ in the cap layer in the hybrid materials. More details for NSL fabrication information are listed in $[1,35]$.

\section{Applications of Hybrid Nanostructures}

There are many applications for the hybrid nanomaterials. They are widely used as polymers [36, 37]. Polymers can be made into various bulk forms and thin films and widely applied to optical and electronic materials, biomaterials, catalysis, sensing, coating, and energy storage, and so forth fields. Here, we only focus on our research groups' applications such as protein A and SEB detection based on our designed nanostructured array.

4.1. Detecting Protein A. The LSPR-based nanobiosensors are extremely sensitive to variation of refractive index (RI) within a few hundred nanometers of gold surface. Capture of the target analyte (Protein A, from Sigma-Aldrich) by the specific reaction between the metal $\mathrm{Au}$ and the Protein A. Protein A bound to the sensing face changes the apparent RI due to solution displacement by the analytes of higher refractive index. To test the detection capability of the hybrid $\mathrm{Au}-\mathrm{Ag}$ nano-biosensors, experiments were performed using solutions of Protein A in PBS buffer $(1: 100,0.01 \mathrm{M}, \mathrm{pH}$ 7.4, from Jinshan Chemical Analyte Pte. Ltd.), and the refractive index of Protein A (1.3352) was detected by Abbe refractometer ZWA-J (temperature $20^{\circ} \mathrm{C}, \Delta n= \pm 0.0002$ ). All the buffer used in the experiments was prepared using double glass-distilled water.

Resonant wavelength $\lambda_{\max }$ of the bare hybrid Au-Ag nanostructures (see Figure 5, black line) was measured to be $575.99 \mathrm{~nm}$. Exposure to $1: 100$ Protein A resolution resulted

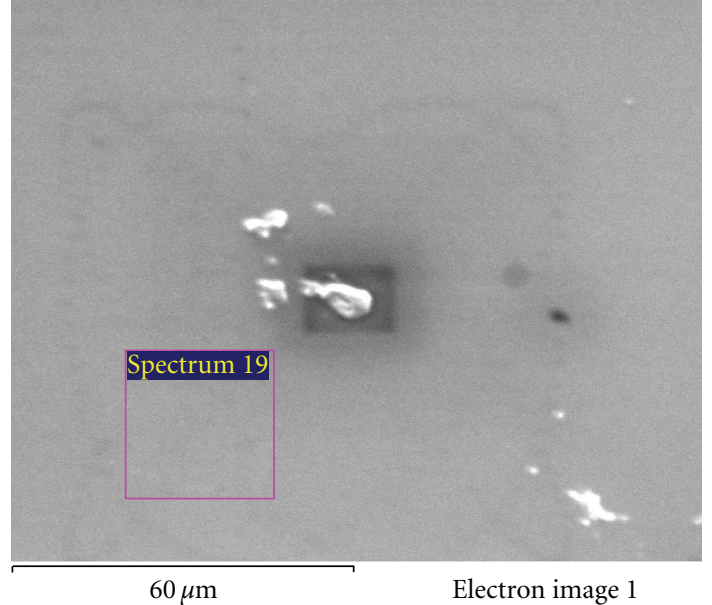

(a)

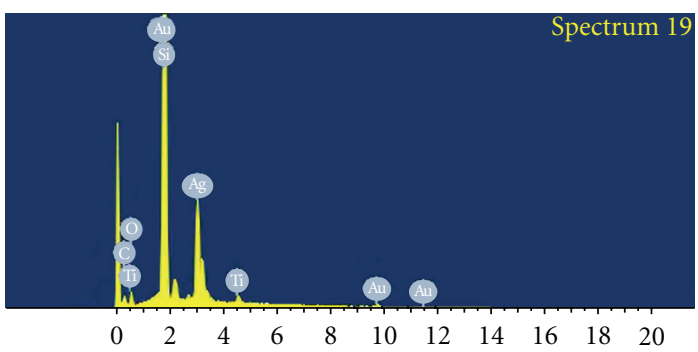

Full scale 8992 cts cursor: 6.061 (129 cts)

$(\mathrm{keV})$

(b)

FIGURE 4: EDX analysis results for hybrid nanostructured array, (a) spectrum area captured by DEX, (b) EDX elements results of hybrid materials.

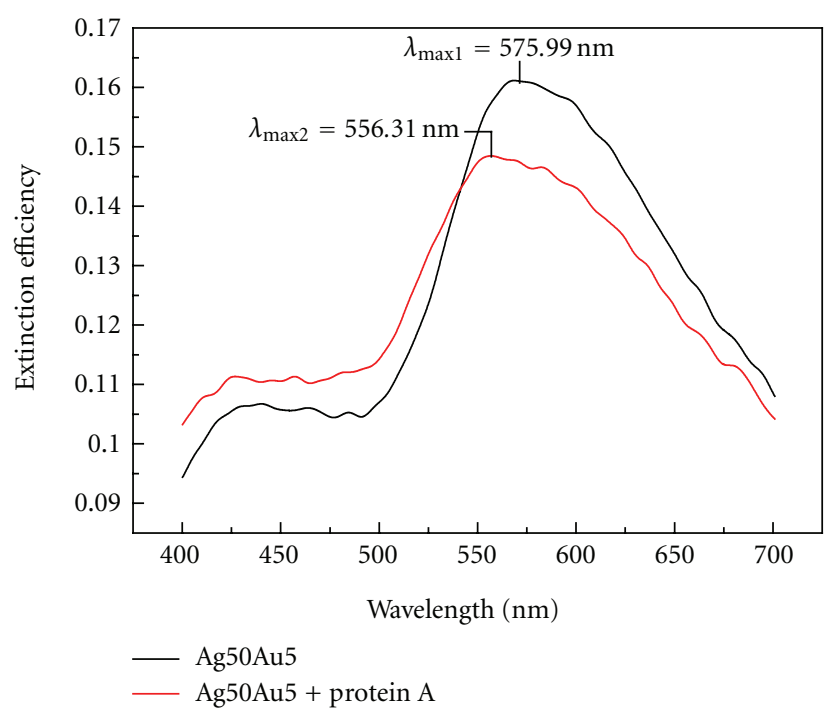

Figure 5: Measured spectra for both pure hybrid Au-Ag (thickness of the $\mathrm{Ag}$ and $\mathrm{Au}$ is $50 \mathrm{~nm}$ and $5 \mathrm{~nm}$, resp.) nanostructure array and binding with protein A. From [1]. 


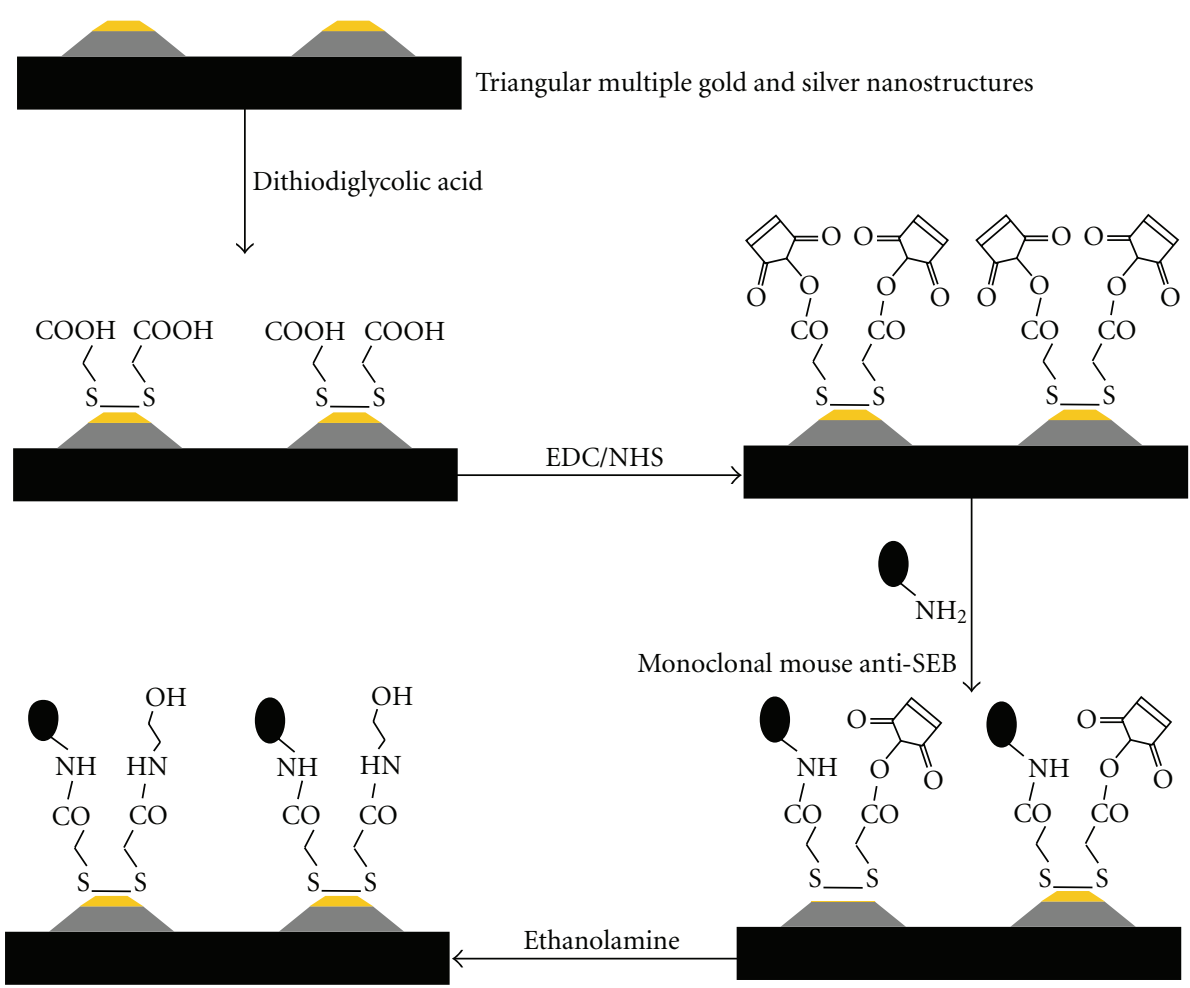

FIGURE 6: Schematic illustration of prefunction for the nanostructure surface. From [2].

in $\lambda_{\max }=556.31 \mathrm{~nm}$ (see Figure 5, red line), corresponding to a $-19.68 \mathrm{~nm}$ shift. It should be noted that $\Delta \lambda_{\max }=$ $-19.68 \mathrm{~nm}$ is smaller than the calculated result of the FDTD. It attributes the experiment defects caused by the NSL fabrication technique. The blue shift is caused by the scattered radiation from the incident radiation $(\omega)$ less than the vibrational frequencies of the molecules $\left(\omega_{\mathrm{vib}}\right)$.

4.2. Detecting SEB. For SEB detection, Our experiments were carried out using home-made SEB prepared by our collaborator (Chinese Academy of Chemical Defence). Monoclonal mouse anti-SEB was purchased from Chemicon; 1Ethyl-3-(3-dimethylaminopropyl) carbodiimide-HCI (EDC) and Sulfo-Nhydroxysuccinimide (S-NHS) from Pierce; ethanolamine from Sigma-Aldrich; dithiodiglycolic acid from Sigma; and phosphate buffer solution (PBS, $0.01 \mathrm{M}$, pH7.4) from Jinshan Chemical analyte Pte. Ltd. The buffer used in the experiments was prepared using double glassdistilled water.

Functionalization of the sensor is a multistep process that prepares the surface for specific detection applications. Prior to each experiment, the triangular hybrid Au-Ag nanostructure array was cleaned and prefunctionalized according to the following protocol: (1) the hybrid nanostructures were cleaned by sonicating the sample in ethanol in $3 \mathrm{~min}$.; (2) dithiodiglycolic acid ( $2 \mathrm{mM})$ aqueous solution was dropped to the surface of the samples and reacting in $30 \mathrm{~min}$.; (3) the carboxyl groups on dithiodiglycolic acid were activated in $30 \mathrm{~min}$. using the same volume of EDC (0.4 M) and S-NHS $(0.1 \mathrm{M})$; (4) the samples were thoroughly rinsed with

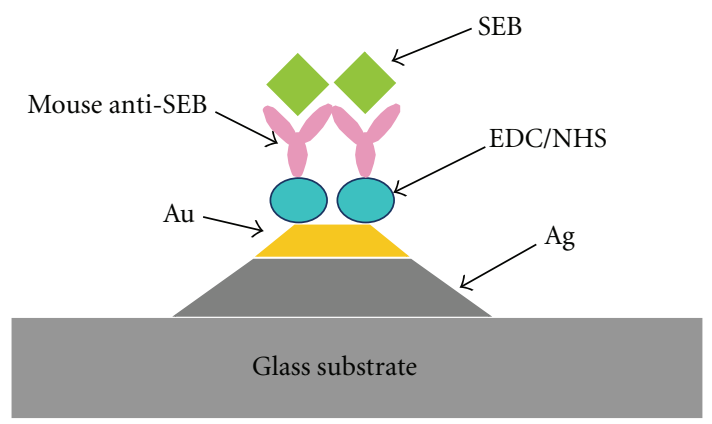

FIGURE 7: Schematic diagram of LSPR-sensor. From [2].

0.01 M PBS buffer and then dried by $\mathrm{N}_{2}$ blowing with highpressure; (5) monoclonal mouse anti-SEB IgG antibodies were coupled to the surface of the nanostructures by dropping $10 \mu \mathrm{g} / \mathrm{mL}$ solution in PBS buffer through chemical reaction between amidogen beside the alkaline aminophenol (Arg and Lys) of IgG and the active carboxyl; (6) redundant active ester groups were enclosed by $1 \mathrm{M}$ ethanolamine aqueous solution. Finally, the samples were rinsed by $0.01 \mathrm{M}$ PBS buffer and a hybrid Au-Ag SEB nano-biosensor was formed. A schematic illustration of the prefunction for the nanostructures surface and a description (with figure) of the LSPR-sensor are shown in Figures 6 and 7, respectively.

The LSPR nano-biosensors are extremely sensitive to a change of refractive index (RI) within the range of a few hundred nanometers from the Au surface. Capturing of the target analyte (SEB) by homologous antibody (monoclonal 


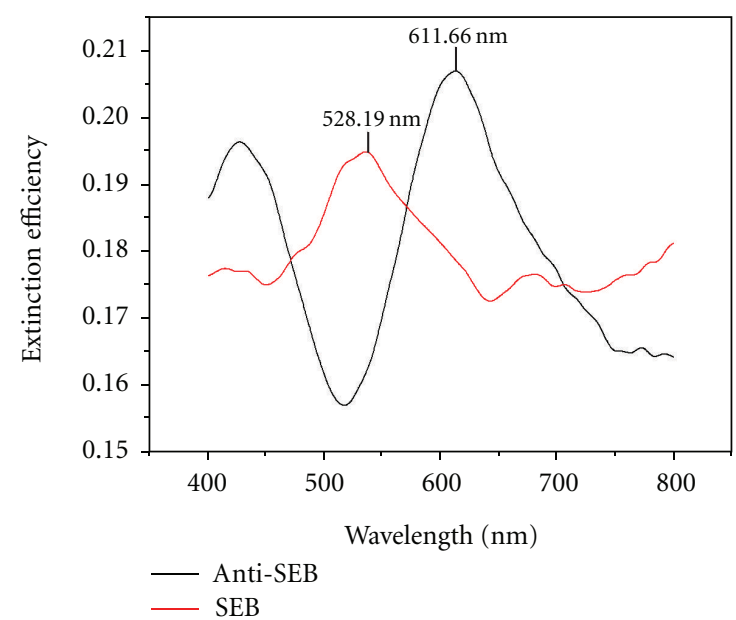

(a)

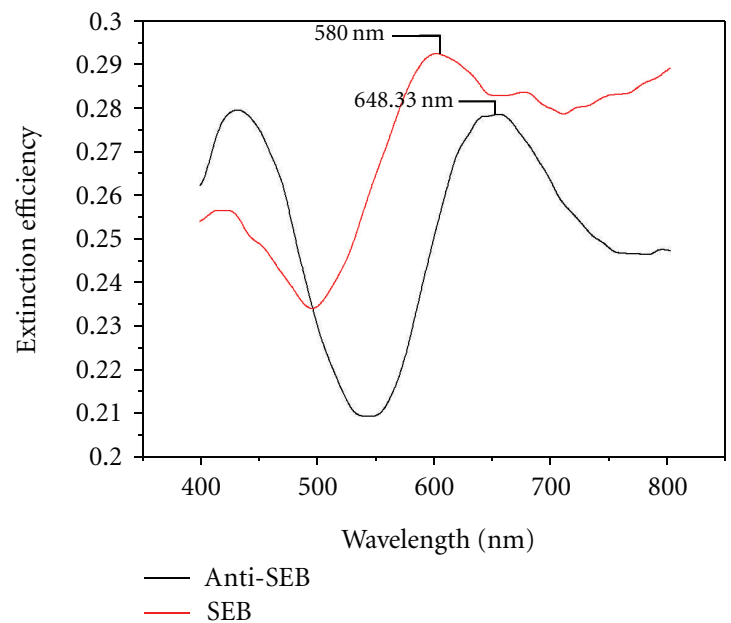

(b)

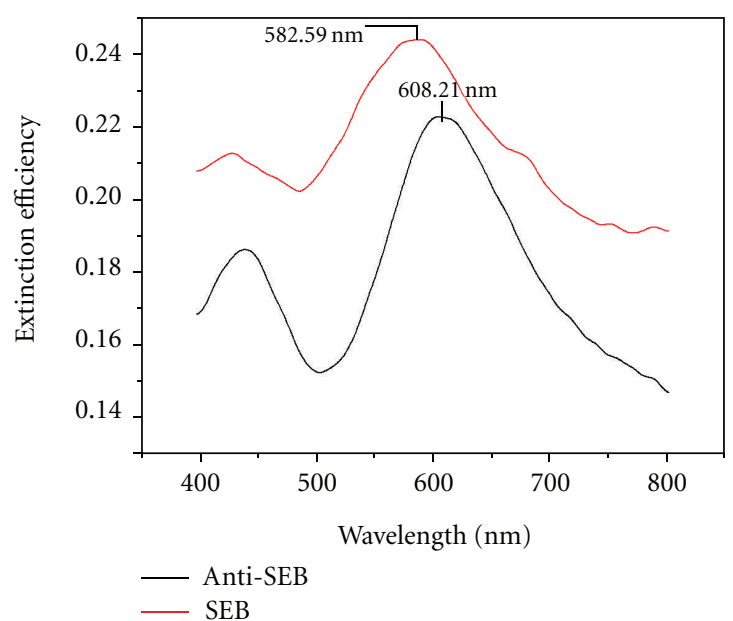

(c)

FIGURE 8: Measured spectra of the LSPR-based hybrid Au-Ag nanobiosensor for specific binding detection with detection concentration of (a) $10 \mu \mathrm{g} / \mathrm{mL}$ SEB, (b) $1 \mu \mathrm{g} / \mathrm{mL}$ SEB, and (c) $100 \mathrm{ng} / \mathrm{mL}$ SEB. From [2].

mouse anti-SEB antibody) bound to the sensing surface varies the RI significantly due to a solution displacement by the analytes with higher refractive index. To test the detection capability of the hybrid Au-Ag triangular particle-based nano-biosensors, experiments were carried out on the basis of the SEB solutions of various concentrations in PBS buffer. Three independent experiments were performed for the SEB solutions in different concentrations of $10 \mu \mathrm{g} / \mathrm{mL}, 1 \mu \mathrm{g} / \mathrm{mL}$, and $100 \mathrm{ng} / \mathrm{mL}$ SEB, respectively. In this study, the LSPR spectra for the specific binding signals were measured using the integrated LSPR biosensor (see Figure 8(a)). The LSPR $\lambda_{\max }$ after monoclonal mouse anti-SEB antibody attachment (see Figure 8(a), black line) was measured to be $611.66 \mathrm{~nm}$. Exposure to $10 \mu \mathrm{g} / \mathrm{mL} \mathrm{SEB}$, corresponding peak wavelength of LSPR $\lambda_{\max }=528.19 \mathrm{~nm}$ (see Figure 8(a), red line), which corresponds to a $\Delta \lambda_{\max }=-83.47 \mathrm{~nm}$ peak shift. Hereinafter, "+" denotes a red-shift and "-" a blue-shift. When the concentration of the SEB solution is changed to be $1 \mu \mathrm{g} / \mathrm{mL}$ and $100 \mathrm{ng} / \mathrm{mL}$, the corresponding peak shift- ing of LSPR is $\Delta \lambda_{\max }=-68.33 \mathrm{~nm}$ (see Figure 8(b)), and $\Delta \lambda_{\max }=-25.62 \mathrm{~nm}$ (see Figure 8(c)), respectively. The main absorbance peak for the anti-SEB drifting from sample to sample (Figures $8(\mathrm{a})-8(\mathrm{c})$ ) attributes to the changing of effective refractive index of the surrounding medium due to binding of SEB and anti-SEB. It should be noted that $\Delta \lambda_{\max }=-25.62 \mathrm{~nm}$ is greatly larger than the resolving power of the integrated LSPR sensor (spectrum resolving power of the spectrometer is $1.7 \mathrm{~nm}$ ). Therefore, it is reasonable to believe that even the SEB solution in lower concentration can be detected by the integrated LSPR biosensor. These will be performed in our next research project. All the extinction measurements were collected at atmosphere environment. To further explore detection performance of the SEB sensors, the concentration of SEB as low as $1 \mathrm{ng} / \mathrm{mL}$ was applied on the biochips. Figure 9 is the measured spectra of the LSPR-based hybrid Au-Ag nano-biosensor for the specific binding detection with detection concentration of $1 \mathrm{ng} / \mathrm{mL}$ SEB. It can be seen that the peak wavelength blue shifts 


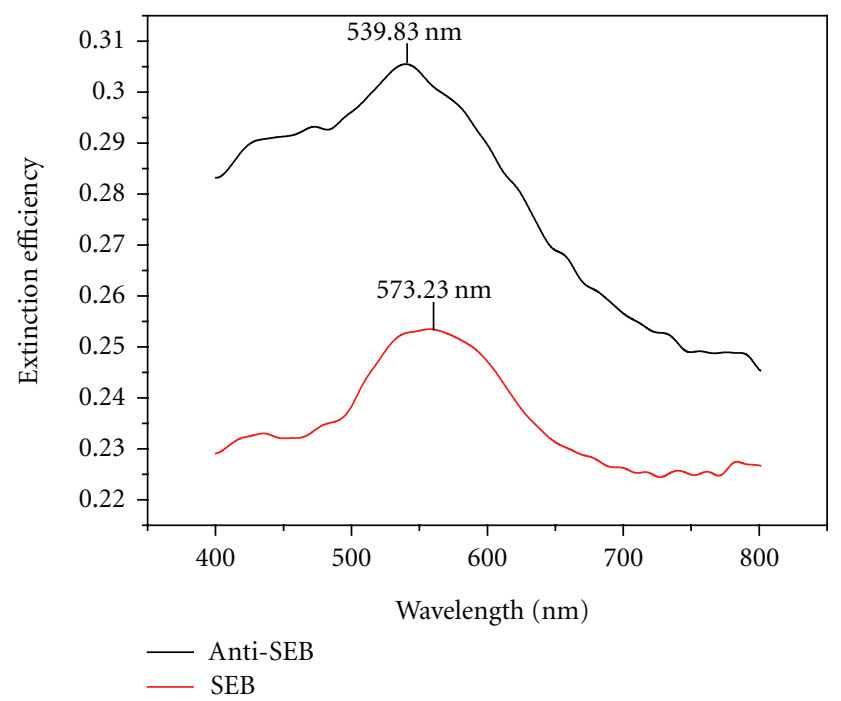

FIGURE 9: Measured spectra of the LSPR-based hybrid Au-Ag nanobiosensor for specific binding detection with detection concentration of $1 \mathrm{ng} / \mathrm{mL}$ SEB. From [2].

are $\Delta \lambda_{\max }=539.83 \mathrm{~nm}-572.23 \mathrm{~nm}=-32.4 \mathrm{~nm}$. Actually, the differences of the resonance peak position are caused by different refractive index materials around the Ag nanostructures. The refractive index of materials changed can influence the resonance peak position. When the concentration of the SEB changed, the peak shifts will change. The red and blue shifts are caused by the scattered radiation from the incident radiation $(\omega)$ by an amount that corresponds to vibrational frequencies of the molecules $\left(\omega_{\mathrm{vib}}\right)$. The frequency emitted by the photo $\omega_{R}=\omega \pm \omega_{\text {vib }}$. If $\omega>\omega_{\text {vib }}$, it causes red shift; if $\omega<\omega_{\text {vib }}$ (smaller SEB concentration), it leads to blue shift.

Our Ag-Au composite structures can be verified by our binding detection results, as shown in Figure 8 . If the structure does not involve $\mathrm{Au}$, dithiodiglycolic acid (see Figure 6) cannot bind with the nanostructures because it binds with Au only [38]. Dithiodiglycolic acid derivatives have a thiol group that reacts with $\mathrm{Au}$ atoms. Therefore, the immobilization process of dithiodiglycolic acid derivatives on the Au surface occurred spontaneously. The pure Ag particles bind with $-\mathrm{OH}$ - only [11]. The binding detection spectra cannot be obtained without the hybrid structure with $\mathrm{Au}$ capped on the Ag particles. Compared to conventional SPR systems, at the same detection level, our presented LSPR-based biosensor is far simpler than the traditional SPR systems (e.g., Biacore system, etc.). The LSPR system is costeffective, small volume, light weight, and portable because some subsystems such as temperature control, pressure control, and precise incident angle control are not necessary. The important advantages of our approach include that: (1) it can provide good selectivity and sensitivity without the labeling process. Our LSPR biosensor assay can detect SEB at approximately $1 \mathrm{ng} / \mathrm{mL}$ rapidly within $1 \mathrm{~min}$; (2) spatial resolution of our detect approach is a single nanostructure while SPR sensors require at least a $10 \times 10 \mu \mathrm{m}$ area for sensing experiments; (3) our LSPR nanobiosensor does not have temperature control, which can reduce the weight and the volume of the detected spectrometric system; (4) The detected spectrometric system costs only $1 / 30$ of the commercialized SPR instruments.

\section{Summary}

We reviewed the design, fabricate, and applications of our proposed hybrid Au-Ag triangular nanostructures. The refractive index sensitivity of hybrid Au-Ag triangular nanostructures is calculated by FDTD method. And we detected the sensitivity in experiment using protein A. This hybrid $\mathrm{Au}-\mathrm{Ag}$ nanostructures are used to detect SEB solution. The detection sensitivity for SEB is nanogramme per milliliter level. All the design and experiment results show that the hybrid nanostructures are useful in the nanobiosensor research field. It has many potential applications in chemical material research fields.

\section{References}

[1] S. L. Zhu, C. L. Du, and Y. Q. Fu, "Hybridization of localized surface plasmon resonance-based Au-Ag nanoparticles," Biomedical Microdevices, vol. 11, no. 3, pp. 579-583, 2009.

[2] S. L. Zhu, C. L. Du, and Y. Q. Fu, "Localized surface plasmon resonance-based hybrid Au-Ag nanoparticles for detection of Staphylococcus aureus enterotoxin B," Optical Materials, vol. 31, no. 11, pp. 1608-1613, 2009.

[3] H. Nakamura, Y. Mogi, T. Akimoto et al., "An enzymechromogenic surface plasmon resonance biosensor probe for hydrogen peroxide determination using a modified Trinder's reagent," Biosensors and Bioelectronics, vol. 24, no. 3, pp. 455460, 2008.

[4] J. Yuan, R. Oliver, J. Li, J. Lee, M. Aguilar, and Y. Wu, "Sensitivity enhancement of SPR assay of progesterone based on mixed self-assembled monolayers using nanoAu particles," Biosensors and Bioelectronics, vol. 23, no. 1, pp. 144-148, 2007. 
[5] A. J. Haes and R. P. van Duyne, "Nanosensors enable portable detectors for environmental and medical applications," Laser Focus World, vol. 39, no. 5, pp. 153-156, 2003.

[6] F. C. Chien, C. Y. Lin, J. N. Yih et al., "Coupled waveguide-surface plasmon resonance biosensor with subwavelength grating," Biosensors and Bioelectronics, vol. 22, no. 11, pp. $2737-$ 2742, 2007.

[7] G. Rong, H. Wang, L. R. Skewis, and B. M. Reinhard, "Resolving Sub-Diffraction limit encounters in nanoparticle tracking using live cell plasmon coupling microscopy," Nano Letters, vol. 8, no. 10, pp. 3386-3393, 2008.

[8] H. Uzawa, K. Ohga, Y. Shinozaki et al., "A novel sugarprobe biosensor for the deadly plant proteinous toxin, ricin," Biosensors and Bioelectronics, vol. 24, no. 4, pp. 923-927, 2008.

[9] C. R. Yonzon, E. Jeoung, S. Zou, G. C. Schatz, M. Mrksich, and R. P. van Duyne, "A comparative analysis of localized and propagating surface plasmon resonance sensors: the binding of Concanavalin A to a monosaccharide functionalized self-assembled monolayer," Journal of the American Chemical Society, vol. 126, no. 39, pp. 12669-12676, 2004.

[10] J. C. Riboh, A. J. Haes, A. D. McFarland, C. R. Yonzon, and R. P. van Duyne, "A nanoscale optical biosensor: real-time immunoassay in physiological buffer enabled by improved nanoparticle adhesion," Journal of Physical Chemistry B, vol. 107, no. 8, pp. 1772-1780, 2003.

[11] A. J. Haes, S. Zou, G. C. Schatz, and R. P. van Duyne, "A nanoscale optical biosensor: the long range distance dependence of the localized surface plasmon resonance of noble metal nanoparticles," Journal of Physical Chemistry B, vol. 108, no. 1, pp. 109-116, 2004.

[12] D. R. Franz, P. B. Jahrling, A. M. Friedlander et al., "Clinical recognition and management of patients exposed to biological warfare agents," Journal of the American Medical Association, vol. 278, no. 5, pp. 399-411, 1997.

[13] T. Yoshida, "Sarin poisoning in Matsumoto, Nagano prefecture," Journal of Toxicological Sciences, vol. 19, no. 3, pp. 85-88, 1994.

[14] T. Suzuki, H. Morita, K. Ono et al., "Sarin poisoning in Tokyo subway," The Lancet, vol. 345, no. 8955, pp. 980-981, 1995.

[15] H. C. Holloway, A. E. Norwood, C. S. Fullerton, C. C. Engel, and R. J. Ursano Jr., "The threat of biological weapons: prophylaxis and mitigation of psychological and social consequences," Journal of the American Medical Association, vol. 278, no. 5, pp. 425-427, 1997.

[16] T. R. Jensen, M. L. Duval, K. L. Kelly, A. Lazarides, G. C. Schatz, and R. P. van Duyne, "Nanosphere lithography: effect of the external dielectric medium on the surface plasmon resonance spectrum of a periodic array of silver nanoparticles," Journal of Physical Chemistry B, vol. 103, no. 45, pp. 9846-9853, 1999.

[17] P. A. Emanuel, J. Dang, J. S. Gebhardt et al., "Recombinant antibodies: a new reagent for biological agent detection," Biosensors and Bioelectronics, vol. 14, no. 10-11, pp. 751-759, 2000.

[18] K. A. Uithoven, J. C. Schmidt, and M. E. Ballman, "Rapid identification of biological warfare agents using an instrument employing a light addressable potentiometric sensor and a flow-through immunofiltration-enzyme assay system," Biosensors and Bioelectronics, vol. 14, no. 10-11, pp. 761-770, 2000.

[19] C. A. Rowe-Taitt, J. P. Golden, M. J. Feldstein, J. J. Cras, K. E. Hoffman, and F. S. Ligler, "Array biosensor for detection of biohazards," Biosensors and Bioelectronics, vol. 14, no. 10-11, pp. 785-794, 2000.
[20] H. Yu, J. W. Raymonda, T. M. McMahon, and A. A. Campagnari, "Detection of biological threat agents by immunomagnetic microsphere-based solid phase fluorogenic- and electrochemiluminescence," Biosensors and Bioelectronics, vol. 14, no. 10-11, pp. 829-840, 2000.

[21] T. O’Brien, L. H. Johnson III, J. L. Aldrich et al., “The development of immunoassays to four biological threat agents in a bidiffractive grating biosensor," Biosensors and Bioelectronics, vol. 14, no. 10-11, pp. 815-828, 2000.

[22] N. N. Alexei, D. S. Scott, K. N. Di et al., "Detection of Staphylococcus aureus enterotoxin $\mathrm{B}$ at femtomolar levels with a miniature integrated two-channel surface plasmon resonance (SPR) sensor," Biosensors and Bioelectronics, vol. 17, no. 6-7, pp. 573-584, 2002.

[23] S. L. Zhu, X. G. Luo, C. L. Du et al., "Hybrid metallic nanoparticles for excitation of surface plasmon resonance," Journal of Applied Physics, vol. 101, no. 6, Article ID 064701, 2007.

[24] S. L. Zhu, C. L. Du, Y. Q. Fu, and A. Liu, "Tuning optical properties of rhombic hybrid $\mathrm{Au}-\mathrm{Ag}$ nanoparticles: a discrete dipole approximation calculation," Journal of Computational and Theoretical Nanoscience, vol. 6, no. 5, pp. 1034-1038, 2009.

[25] S. L. Zhu and W. Zhou, "Effect of gold coating on sensitivity of rhombic silver nanostructure array," Plasmonics, vol. 4, no. 4, pp. 303-306, 2009.

[26] S. L. Zhu and W. Zhou, "Optical characteristics of rhombic hybrid au-ag nanostructures calculated by discrete dipole approximation method," Journal of Computational and Theoretical Nanoscience, vol. 7, no. 3, pp. 634-637, 2010.

[27] S. L. Zhu and W. Zhou, "Sensitivity of triangular hybrid Au-Ag nanostructure array," Journal of Computational and Theoretical Nanoscience, vol. 7, no. 8, pp. 1414-1517, 2010.

[28] A. L. Aden and M. Kerker, "Scattering of electromagnetic waves from two concentric spheres," Journal of Applied Physics, vol. 22, no. 10, pp. 1242-1246, 1951.

[29] B. T. Draine and P. J. Flatau, "Discrete-dipole approximation for scattering calculations," Journal of the Optical Society of America A, vol. 11, no. 4, pp. 1491-1499, 1994.

[30] A. Skarlatos, R. Schuhmann, and T. Weiland, "Solution of radiation and scattering problems in complex environments using a hybrid finite integration technique-uniform theory of diffraction approach," IEEE Transactions on Antennas and Propagation, vol. 53, no. 10, pp. 3347-3357, 2005.

[31] C. Oubre and P. Nordlander, "Optical properties of metallodielectric nanostructures calculated using the finite difference time domain method," The Journal of Physical Chemistry B, vol. 108, no. 46, pp. 17740-17747, 2004.

[32] A. A. Pisano, A. Sofi, and P. Fuschi, "Finite element solutions for Eringen-type nonlocal elastic 2D problems," International Journal of Solids and Structures, vol. 46, no. 21, pp. 3836-3849, 2009.

[33] S. Kawata, M. Ohtsu, and M. Irie, Nano-Optics, Springer, Heidelberg, Germany, 2002.

[34] A. J. Haes and R. P. van Duyne, "A nanoscale optical biosensor: sensitivity and selectivity of an approach based on the localized surface plasmon resonance spectroscopy of triangular silver nanoparticles," Journal of the American Chemical Society, vol. 124, no. 35, pp. 10596-10604, 2002.

[35] S. Zhu, C. Du, and Y. Fu, "Fabrication and characterization of rhombic silver nanoparticles for biosensing," Optical Materials, vol. 31, no. 6, pp. 769-774, 2009.

[36] Z. Merican, T. L. Schiller, C. J. Hawker, P. M. Fredericks, and I. Blakey, "Self-assembly and encoding of polymer-stabilized 
gold nanoparticles with surface-enhanced Raman reporter molecules," Langmuir, vol. 23, no. 21, pp. 10539-10545, 2007.

[37] K. J. Thurecht, I. Blakey, H. Peng et al., "Functional hyperbranched polymers: toward targeted in vivo 19F magnetic resonance imaging using designed macromolecules," Journal of the American Chemical Society, vol. 132, no. 15, pp. 53365337, 2010.

[38] M. Lee, Y. Hur, T. Kim et al., "Surface plasmon resonance study on the interaction between the fructose and phenylboronic acid monolayer," Microchemical Journal, vol. 72, no. 3, pp. 315-321, 2002. 

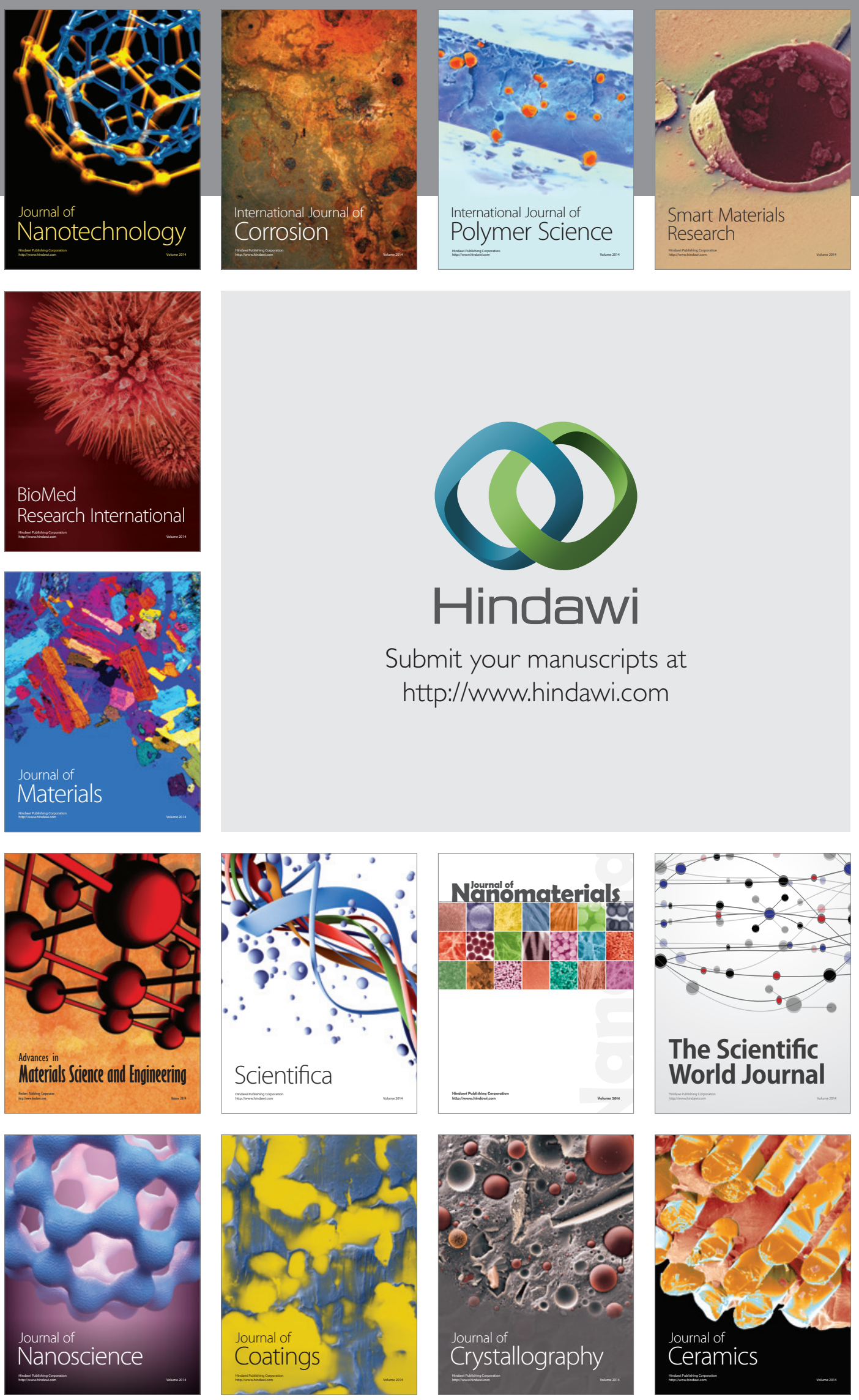

The Scientific World Journal

Submit your manuscripts at

http://www.hindawi.com

\section{World Journal}

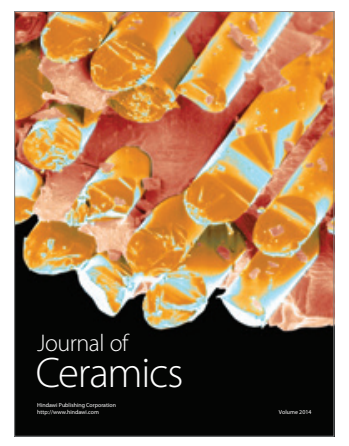

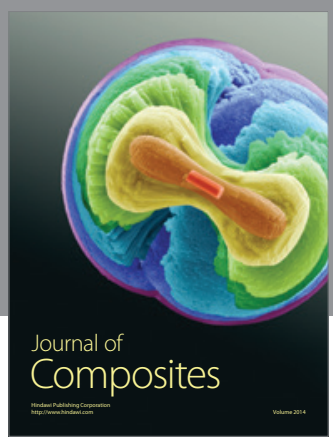
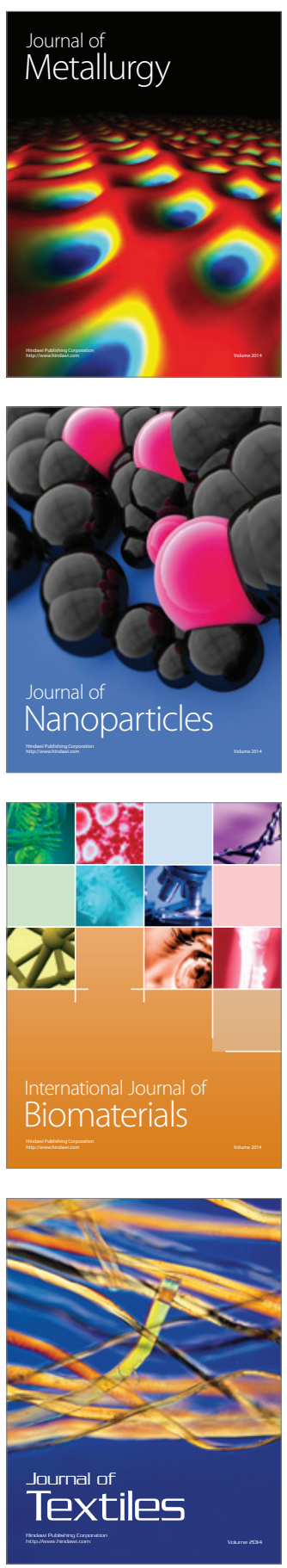